\title{
WORKER PERFORMANCE AND VENTILATION: ANALYSES OF INDIVIDUAL DATA FOR CALL-CENTER WORKERS
}

\author{
CC Federspiel ${ }^{1}, \mathrm{G} \mathrm{Liu}^{2}, \mathrm{M} \mathrm{Lahiff}^{3}$, D Faulkner ${ }^{4}$, DL Dibartolomeo ${ }^{4}$, WJ Fisk ${ }^{4}$, PN Price ${ }^{4}$, \\ DP Sullivan ${ }^{4}$ \\ ${ }^{1}$ Center for the Built Environment, University of California, Berkeley, CA, USA \\ ${ }^{2}$ Department of Electrical Engineering, University of California, Berkeley, CA, USA \\ ${ }^{3}$ School of Public Health, Div. of Biostatistics, University of California, Berkeley, CA, USA \\ ${ }^{4}$ Indoor Environment Dept., Lawrence Berkeley National Laboratory, Berkeley, CA, USA
}

\begin{abstract}
We investigated the relationship between ventilation rates and work performance in a call center. We randomized the ventilation controls and measured ventilation rate, differential carbon dioxide $\left(\Delta \mathrm{CO}_{2}\right)$ concentration, temperature, humidity, occupant density, degree of under-staffing, shift length, time of day, and time required to complete two different work performance tasks (talk and wrap-up). $\Delta \mathrm{CO}_{2}$ concentrations ranged from 13 to $611 \mathrm{ppm}$. We used multi-variable regression to model the association between the predictors and the responses. We found that agents performed talk tasks fastest when the ventilation rate was highest, but that the relationship between talk performance and ventilation was not monotone. We did not find a statistically significant association between wrap-up performance and ventilation. At high temperatures agents were slower at both the talk and wrap-up tasks. Agents were slower at wrap-up during long shifts and when the call center was under-staffed.
\end{abstract}

\section{INDEX TERMS}

Ventilation rates, Productivity, Offices, Worker performance, Carbon dioxide

\section{INTRODUCTION}

This paper describes part two of a two-part analysis from a productivity study performed in a call center operated by a health maintenance organization. The companion paper (Fisk et al., 2002) describes the analysis of group performance data that has good time resolution. The objective of this paper is to report the on the analysis of individual performance data acquired from the same experiment. The analysis described in this paper confirms the findings in the companion paper and reveals additional insight into the relationship between environmental factors and work performance.

\section{METHODS}

The study design and procedures are described in the companion paper (Fisk et al., 2002). In this section we describe methods that are specific to the analysis of the individual performance data.

The call center is an "inbound" call center, which means that calls originate from clients. Calls are forwarded to agents by an automated call distribution (ACD) system. The task of processing a call is referred to as "handling" a call. Handling a call involves two subtasks referred to as "talk" and "wrap-up." The talk task involves the period of time when the agent 
is talking with the client. Wrap-up involves performing tasks such as database entry after the agent has finished talking with the client. When wrap-up is complete the agent signals the ACD system that he is ready to accept another call.

Two kinds of agents work in the call center, registered nurses (RNs) who provide medical advice and tele-service representatives (TSRs) who screen calls and schedule appointments. In this paper we only consider data related to the RNs.

The ACD system measures the duration of every talk task and every wrap-up task. Agents sign in to the ACD system with a unique identification number. We analyzed data from reports showing the average talk time and wrap-up time during each shift for each agent coded by the agent identification number. Individual performance per task or over shorter time intervals than a whole shift were not available. Our response variables were the natural logarithm of the shift-averaged talk times and wrap-up times.

Agents sign out of the ACD system when they finish a shift. We used reports with sign-in and sign-out times for each agent each day to determine the length of each agent's shift.

The ACD system records a metric called NETS. NETS is the number of agents scheduled minus the number needed to keep the waiting time in the queue equal to a target. When NETS is negative the call center is under-staffed.

The call center is organized into clusters of cubicles called "neighborhoods." Most agents work in the same neighborhood each shift. As reported in Fisk et al. (2002), we recorded space temperature and humidity continually during the study in each neighborhood. By associating agents with neighborhoods we were able to associate individual temperature and humidity readings with agents.

The call center has four air-handling units of approximately equal size. For each air-handling unit we recorded the difference between the carbon dioxide concentration in the return duct and the outdoors $\left(\Delta \mathrm{CO}_{2}\right)$. We also determined the outdoor airflow rate based on supply airflow and $\mathrm{CO}_{2}$ concentrations in the return, outdoor, and supply ducts. We associated the $\Delta \mathrm{CO}_{2}$ concentrations and outdoor airflow rates with individual agents by using floor plans to determine which neighborhoods each air-handling unit served.

We computed shift-averaged NETS, temperature, humidity, $\Delta \mathrm{CO}_{2}$, and ventilation rate for each worker. The averages were used as predictors in our models. We used multi-variable regression to model the association between the response variables and the predictors. The ACD system records the number of calls per shift per agent. We used the number of calls as weights in our regression analysis. Our models are multiplicative. The logarithm of the talk time or wrap-up time is modeled as a linear combination of functions of the response variables.

The call center operates 24 hours per day, 7 days per week. We only analyzed data associated with shifts starting after 6am and finishing before $10 \mathrm{pm}$, Monday-Friday. We removed obvious outliers manually. The software that runs the ACD system was upgraded 9 days before the start of the study and on the $58^{\text {th }}$ day of the study (a Friday evening). We observed a transient in the daily-averaged wrap-up times during the first two weeks of the study, so we 
discarded the first day (a Friday) and the following two weeks of data. We found that the transient in the daily-average wrap-up times was shorter after the second software upgrade, so we only discarded one week of data after that event. We included an indicator variable to account for the performance difference after the second software upgrade.

\section{RESULTS}

Descriptive statistics for the explanatory variables are shown in Table 1. The ventilation rate distribution has three modes that correspond to low, medium, and high damper settings. The temperature distribution has a long high-temperature tail. The relative humidity has a long low-humidity tail. The number of occupants includes TSRs because occupant density is a proxy for auditory and visual distractions. The distribution of shift length has four modes corresponding to 4, 5, 6, and 8 hour shifts. Below the 4-hour mode there is a long tail of short shifts.

Table 1: Descriptive statistics of explanatory variables.

\begin{tabular}{|l|l|l|l|l|l|l|l|}
\hline & $\Delta \mathrm{CO}_{2}$ & Vent & Temp & RH & Occ. & NETS & Shift \\
\hline min & $13 \mathrm{ppm}$ & $0.26 \mathrm{l} / \mathrm{s}-\mathrm{m}^{2}$ & $21.0 \mathrm{C}$ & $20.2 \%$ & 66.6 & -11 & $0.48 \mathrm{hrs}$ \\
\hline med & 243 & 1.38 & 23.0 & 42.5 & 156.3 & 1.8 & 6.4 \\
\hline mean & 253 & 2.07 & 23.1 & 42.4 & 159.6 & 2.7 & 6.8 \\
\hline max & 611 & 10.0 & 26.6 & 55.3 & 253.6 & 17.2 & 12.4 \\
\hline
\end{tabular}

We tried models with linear and categorical terms for $\Delta \mathrm{CO}_{2}$, a linear $\Delta \mathrm{CO}_{2}$ term multiplied by shift length, linear, inverse linear, and categorical terms for ventilation rate, ventilation rate multiplied by shift length, quadratic and categorical terms for temperature, quadratic terms for enthalpy, a linear term for occupant density, quadratic and categorical terms for NETS, and linear and categorical terms for shift length. The following results are for a model with a categorical term for either $\Delta \mathrm{CO}_{2}$ or ventilation rate, a categorical temperature term, a linear occupant density term, a categorical NETS term, and a categorical shift length term.

Figures 1 and 2 show the predicted association between talk time and the $\Delta \mathrm{CO}_{2}$ and ventilation categories. Positive "effects" mean the agents worked slower. In both cases, the highest ventilation rate (lowest $\Delta \mathrm{CO}_{2}$ ) is the baseline. Vertical bars in these and all successive figures are standard errors. Boundaries for the $\Delta \mathrm{CO}_{2}$ categories are at 100, 250, and $420 \mathrm{ppm}$. The $\Delta \mathrm{CO}_{2} \mathrm{p}$-values are $0.012,0.15$, and 0.60 . The boundaries for the ventilation categories are $0.76,2.4$, and $4.41 / \mathrm{s}-\mathrm{m} 2$. The ventilation rate p-values are $0.32,0.047$, and 0.022 . 


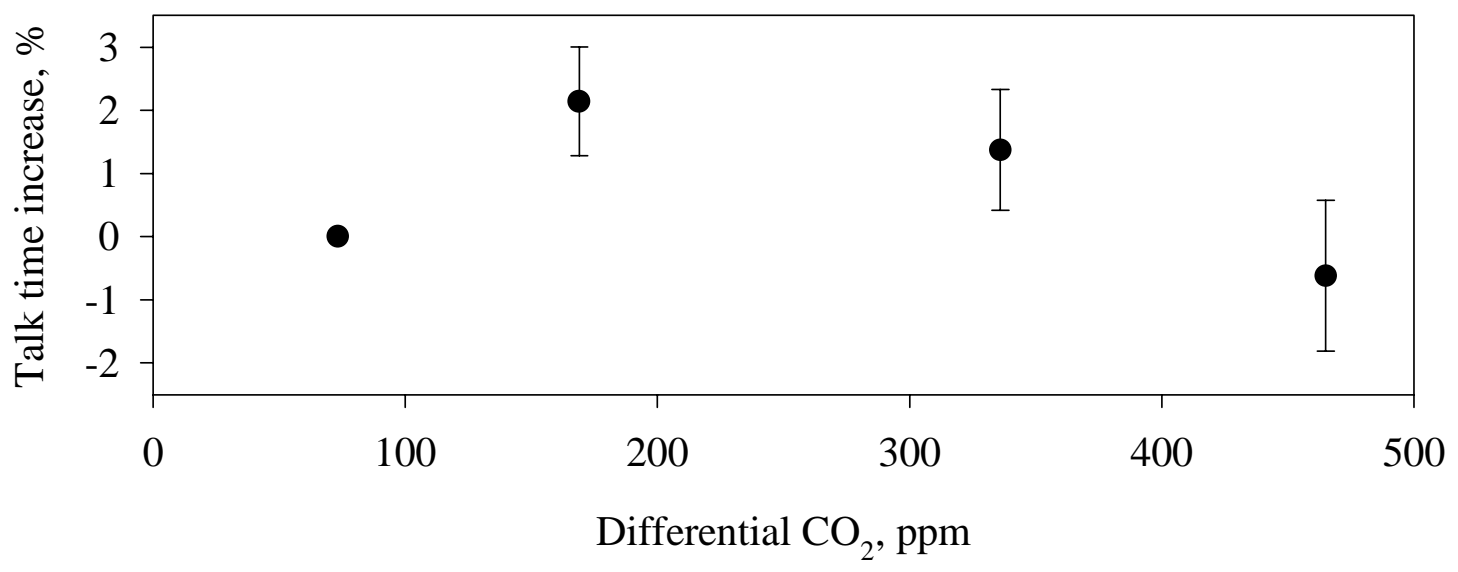

Figure 1: $\Delta \mathrm{CO}_{2}$ category coefficients for talk model.

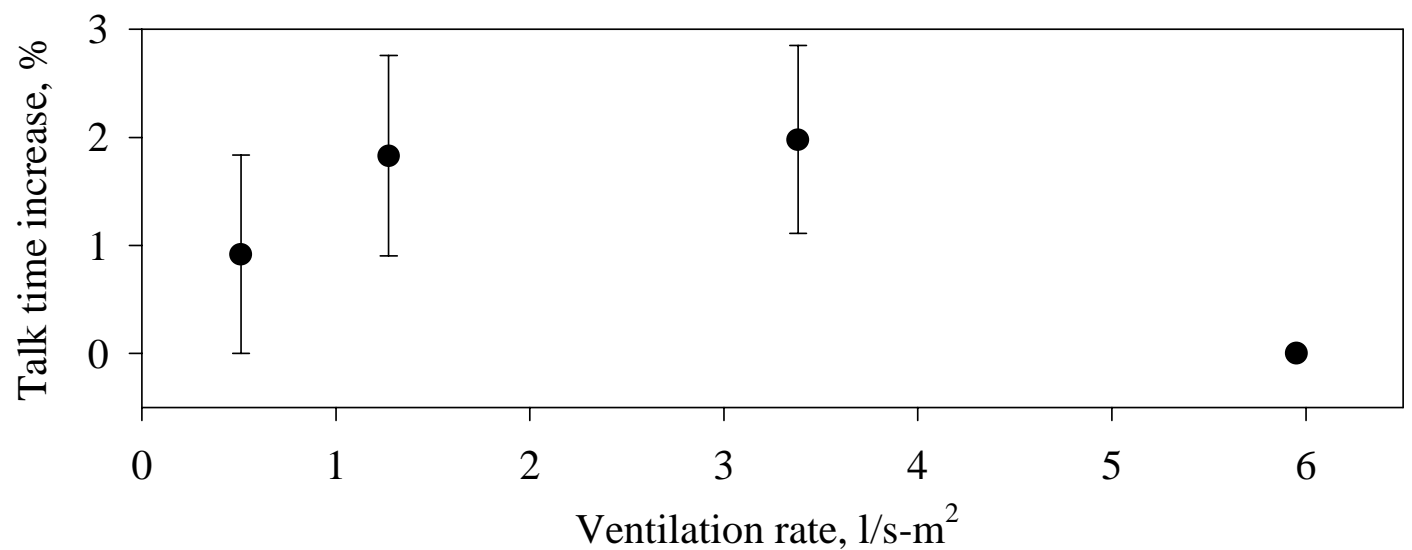

Figure 2: Ventilation category coefficients for talk model.

The model with $\Delta \mathrm{CO}_{2}$ categories predicts that agents take $8.5 \%$ longer to talk at maximum occupancy than at minimum occupancy $(\mathrm{p}=0.05)$. The model with ventilation categories predicts that they take $4 \%$ longer $(\mathrm{p}=0.36)$. The models predicts that agents performed the talk task $4.0 \%$ and $4.3 \%$ slower after the software upgrade $(\mathrm{p}=0)$.

The models of wrap-up time showed no statistically significant association between wrap-up time and differential $\Delta \mathrm{CO}_{2}$ or ventilation rate. They did show an association between wrap-up time and temperature, NETS, and shift length.

Figure 3 shows the coefficients of the temperature categories for the wrap-up model containing $\Delta \mathrm{CO}_{2}$ categorical variables. The baseline is $23^{\circ} \mathrm{C}$. The boundaries between the categories are $21.7,22.5,23.5,24.5$, and $25.4^{\circ} \mathrm{C}$. The p-values for the coefficients are 0.89 , $0.26,0.91,0.98$, and 0.0028 . The model with ventilation rate categories also predicts lower performance at high temperature. 


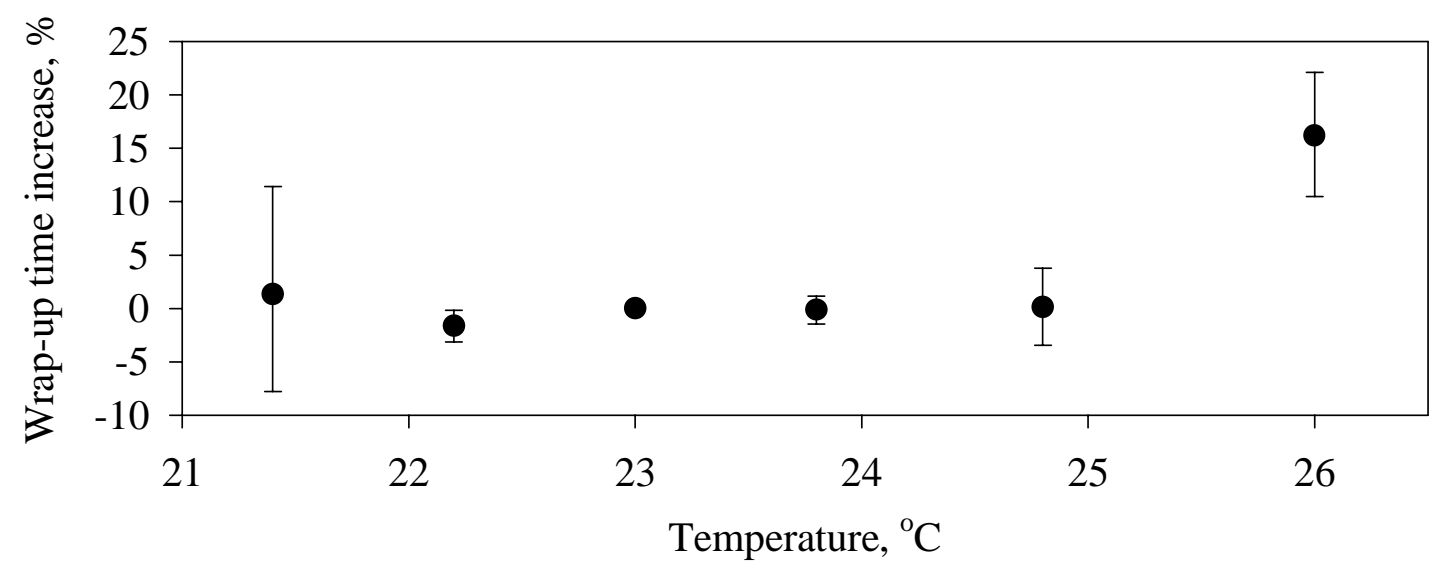

Figure 3: Temperature coefficients for wrap-up model with $\Delta \mathrm{CO}_{2}$.

Figure 4 shows the coefficients of the NETS categories for the same wrap-up model. The baseline is NETS $=0$, meaning no under-staffing or over-staffing. There is a clear trend showing that agents perform wrap-up fastest when NETS is positive (call center overstaffed). The p-values for the NETS coefficients are 0.046, 0.12, 0.30, 0.11, and 0.55.

Figure 5 shows the coefficients of the shift length categories for the same wrap-up model. There is a clear, increasing trend. All of the coefficients less than the baseline (nominal 8-hour shift) are less than zero while the coefficient greater than the baseline is greater than zero. The $\mathrm{p}$-values for the coefficients are 0.060, 0.72, 0.57, 0.0070, and 0.23.

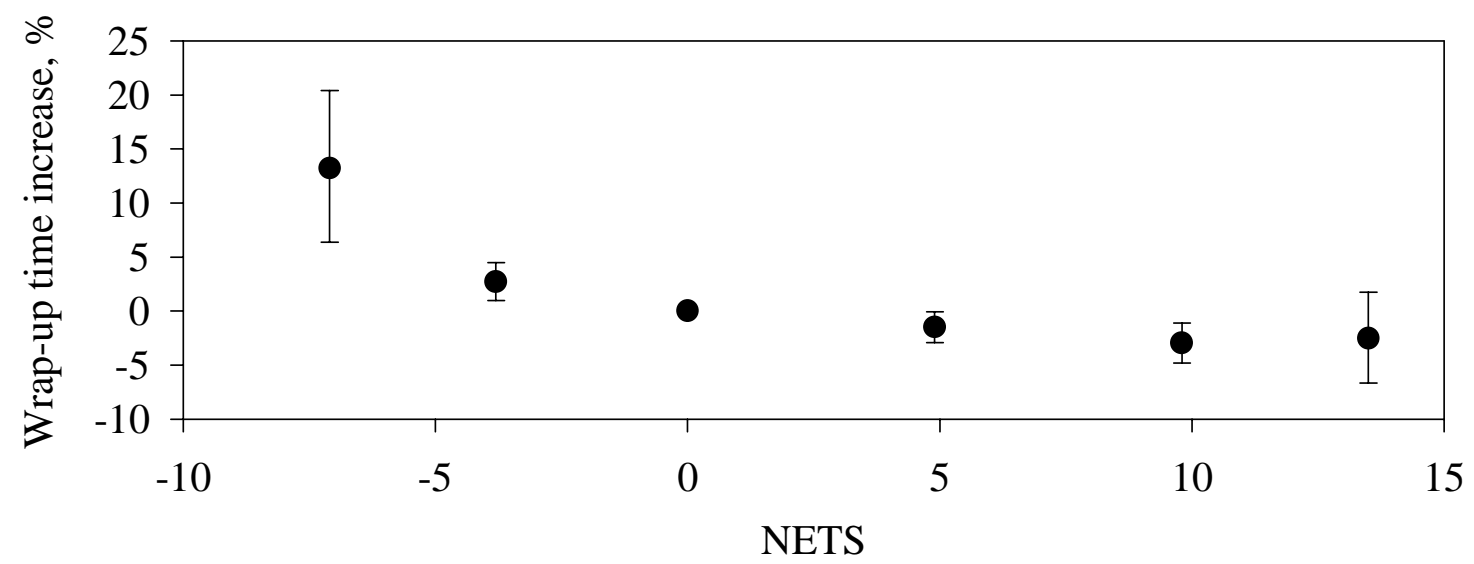

Figure 4: NETS coefficients for wrap-up model with $\Delta \mathrm{CO}_{2}$. 


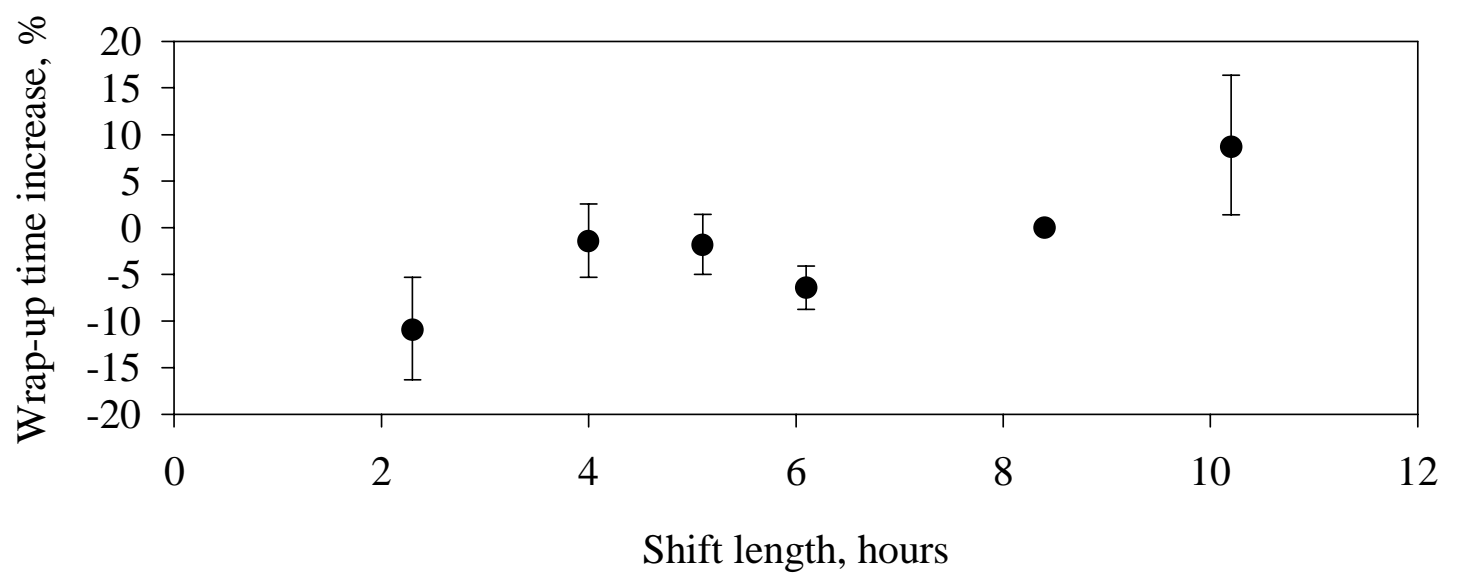

Figure 5: Shift length coefficients for wrap-up model with $\Delta \mathrm{CO}_{2}$.

The wrap-up model with $\Delta \mathrm{CO}_{2}$ categories predicts that wrap-up is $19.7 \%$ longer at full occupancy than at minimum occupancy $(\mathrm{p}=0.067)$. The same model predicts that wrap-up was $7.5 \%$ shorter after the software upgrade $(\mathrm{p}=0)$.

\section{DISCUSSION}

We expected, but did not find, a dose-response relationship between ventilation and work performance. Instead we found that performance at low and high ventilation rates was statistically the same, that performance at intermediate ventilation rates was worse by about $2 \%$, at that the increase was statistically significant. We tried a number of different models, including models containing the air quality proxies multiplied by shift length (time of exposure). The pattern described above showed up in most of these models.

We found that what evidence there was of an association between ventilation and performance showed up in the talk task and not in the wrap-up task. We expected that productivity loss would be more likely during wrap-up because agents are free to work at their own pace; they are not constrained by their interaction with a client.

We found that agents were $16 \%$ slower at wrap-up when the temperature was greater than $25.4{ }^{\circ} \mathrm{C}$. This was the largest effect with a p-value less than $5 \%$. However, temperatures greater than $25.4{ }^{\circ} \mathrm{C}$ occurred on only seven days, six of them in a nine-day period starting at day 21 . Some models we tried showed a similar increase in wrap-up times at low temperatures, but the low-temperature terms were never statistically significant.

When the call center was understaffed agents performed wrap-up slower. The call center director predicted this effect prior to our analysis. The hypothesis is that when the call center is understaffed agents never get to rest between calls. They make up for the missing rest by working slower during wrap-up.

\section{CONCLUSION AND IMPLICATIONS}

1. There is some evidence that ventilation rates less than $100 \%$ outdoor air are associated with lower work performance, but the results are not conclusive.

2. There is some evidence that high temperature $(>25.4 \mathrm{C})$ is associated with lower work performance. 
3. There is some evidence that higher occupant density is associated with lower work performance.

4. Understaffing is associated with lower work performance.

5. Longer shifts are associated with lower work performance.

\section{ACKNOWLEDGEMENTS}

This work was supported by the Center for the Built Environment at U.C. Berkeley and by the Assistant Secretary for Energy Efficiency and Renewable Energy, Office of Building Technology, State, and Community Programs, Office of Building Research and Standards of the U.S. Department of Energy (DOE) under contract No. DE-AC03-76SF00098.

\section{REFERENCES}

Fisk, WJ, PN Price, D Faulkner, DP Sullivan, DL Dibartolomeo, CC Federspiel, G Liu, and M Lahiff, 2002, "Worker Performance and Ventilation: Analyses of Time-Series Data for a Group of Call Center Workers," Indoor Air 2002, Monterey, CA, June 30 - July 5. 